\title{
Doppler Ultrasound Studies of the Ophthalmic Artery
}

\author{
C. R. CANNING and M. RESTORI \\ London
}

\begin{abstract}
Summary
The doppler frequency shift of ultrasound pulses scattered off red blood cells in the ophthalmic artery can be detected and used as an index of velocity of flow in the artery. The doppler shift is shown to be responsive to changes in ocular blood flow induced by changes in mean arterial blood pressure at the level of the eye and changes in intraocular pressure. The technique may be useful in the study of eye disease in which blood flow is altered. Doppler frequency shifted signals have also been detected within the coats of the eye.
\end{abstract}

The doppler frequency shift in ultrasonic pulses has been used for many years to assess flow rate in blood vessels in vivo. The technique has until recently been limited by not being able to measure simultaneously both the doppler signal and visualise its source in 'real-time'. Recent technological advances make it possible to select a region within a 'real-time' B-mode image and obtain doppler frequency shift information from this selected region at the same time.

The preliminary studies reported here were designed to determine whether a reliable noninvasive index of velocity of blood flow to the eye could be measured.

The ophthalmic artery is a small vessel arising from the internal carotid artery. It has a variable course and inconstant branching pattern. ${ }^{1}$ Only a proportion of the blood in the artery supplies the eye; the rest supplies the nose, ethmoid sinuses, lacrimal gland and forehead. These vessels are too small to be seen directly on a B-mode image. Their relation to the ophthalmic artery is however known. It is necessary to demonstrate that a doppler signal obtained behind the globe is specifically responsive to changes in blood flow within the eye rather than to flow within the other structures supplied by the ophthalmic artery.

The region most likely to contain the pos- terior ciliary arteries which supply the eye, and with the least chance of including branches to non-ocular structures, is close to the optic nerve and as close to the globe as possible. This is the region which has been studied for doppler signals.

Most of the blood flow to the eye supplies the choroid. There is no autoregulation in the choroidal circulation ${ }^{2}$ so changes in either mean arterial blood pressure (BP) or intraocular pressure (IOP) produce a linear response in ocular blood flow. These two factors (BP, IOP) have been varied independently to determine whether the doppler frequency shift recorded behind the globe is responsive to the predicted changes in ocular circulation.

\section{Methods}

Equation 1 describes the relationship between the doppler shifted frequency $f_{D}$ (the difference between the incident and returned frequency after reflection of an ultrasonic pulse) and the velocity of blood flow.

$$
f_{D}=-\frac{2 f v \cos \theta}{c} \ldots \ldots \ldots . \text { Equation }(1)
$$

where $f_{D}=$ Doppler shifted frequency

$$
\begin{aligned}
f= & \text { incident frequency of the } \\
& \text { interrogating pulse } \\
\mathbf{v}= & \text { velocity of the scatterer }
\end{aligned}
$$


$\theta=$ angle between the transducer axis and the direction of flow of the scatterer

c = velocity of sound in the transmitting medium

Figure 1 shows this diagrammatically. The red blood cells, the ultrasonic scatterers in blood, move with a range of velocities and in practice the doppler frequency signal obtained is complicated.

The system used, the Acuson 128, ${ }^{*}$ employs an array of 128 transducers. Shown in Figure 2 is a B-mode image, beneath which is the doppler spectrogram from a selected region. The dotted line indicates the direction

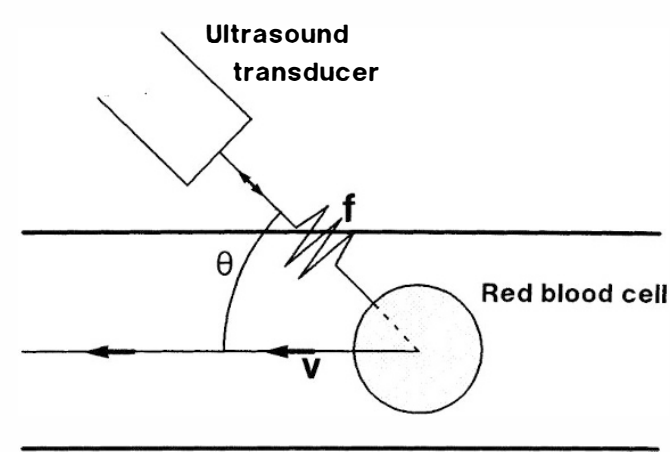

Blood vessel

Fig. 1. Doppler effect: a single scatterer moving towards the interrogating pulse.

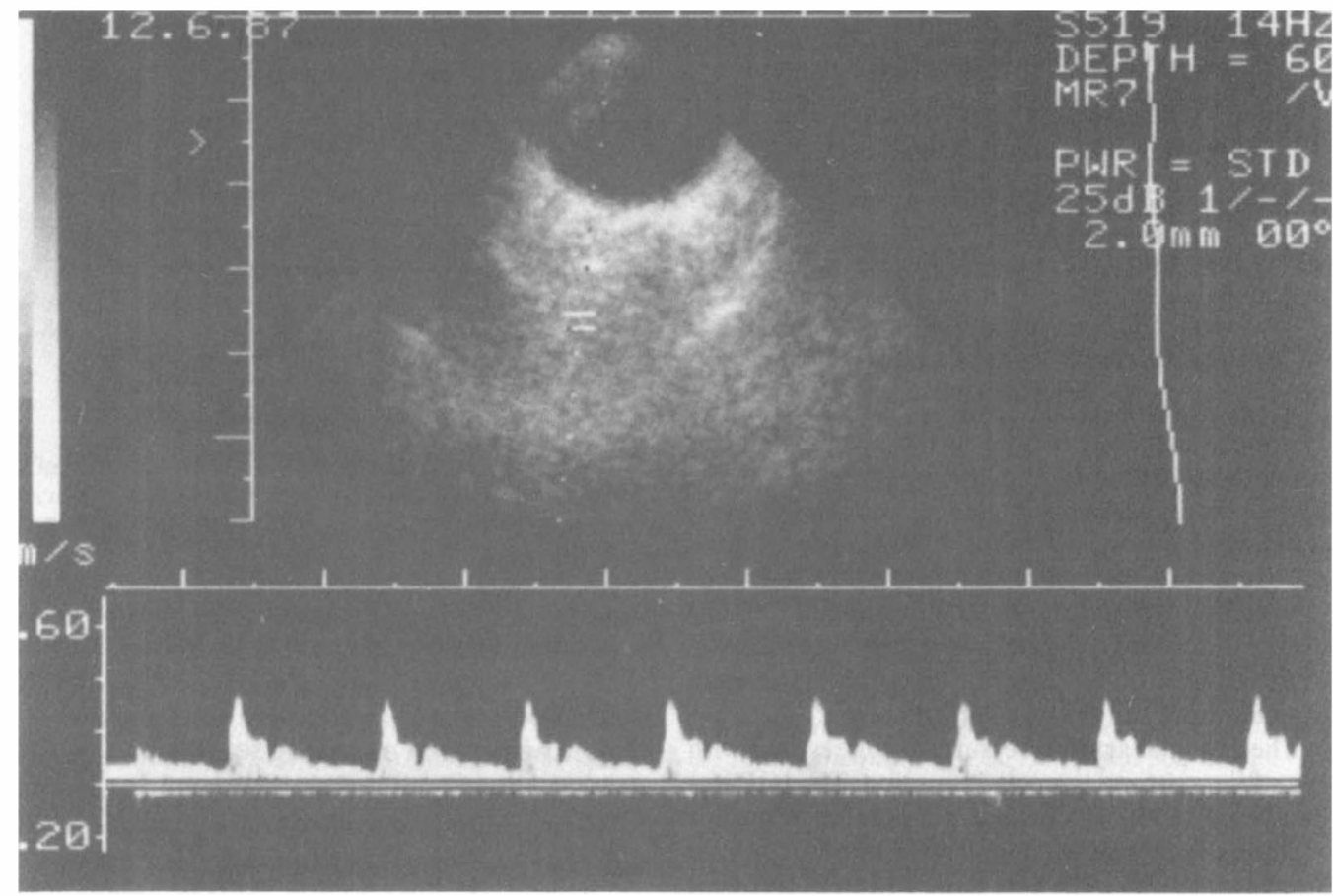

Fig. 2. B-mode image with doppler spectrogram beneath. The doppler frequency shift is proportional to velocity, so the $Y$-axis is scaled in $\mathrm{ms}^{-1}$.

of travel of interrogating ultrasonic pulses and the two cursors, forming the gate, the region from within which echoes are selected for doppler study. Here the gate is positioned alongside and inferonasal to the optic nerve approximately $15 \mathrm{~mm}$ behind the globe. The spectrogram is a plot of doppler shifted fre-

*Acuson Computed Sonography Model 128 plus $5 \mathrm{mHz}$ sector transducer (Acuson Computed Sonography, Bessemer Drive, Stevenage, Herts SG1 2DX). quency against time. By convention, blood flow towards the transducer is displayed above the axis and away from the transducer beneath the axis. The transducer is moved and angled to maximise both the volume and the pitch of the audible doppler shifted frequency. This implies that the vessel being measured is pointing as much as possible along the pathway of the interrogating pulses-angle $\theta$ is minimised. 
It is never possible to be sure that angle $\theta$ is zero; only the vector component of velocity along the direction of travel of the ultrasonic pulses can be measured. For this reason the absolute velocity displayed on the spectrogram cannot be used as a basis for comparison between different eyes. Certain characteristics of the shape of the spectrogram, expressed as ratios to eliminate angle $\theta$, are generally used for such comparisons. Two possible ratios are described in equations 2 and 3.

Resistivity Index $(\mathrm{RI})=\frac{\mathrm{S}-\mathrm{D}}{\mathrm{S}} \ldots \ldots$ Equation (2)

Pulsatility Index $(\mathrm{PI})=\frac{\mathrm{S}-\mathrm{D}}{\mathrm{T}(\mathrm{A}) \max } \ldots$ Equation (3)

where $S=$ maximum systolic velocity

$\mathrm{D}=$ minimum diastolic velocity

$\mathrm{T}(\mathrm{A}) \max =$ average height of the

spectrogram over several heart beats (the time averaged mean of the maximum velocity)

\section{Study 1: Posture}

The mean arterial pressure at the level of the eye depends upon the height of the eye above the heart. Lying, the eye and heart are on the same level; standing or sitting raises the eye about $30 \mathrm{~cm}$ above the heart-a pressure drop of $22 \mathrm{mmHg}$. Ophthalmodynamometry measurements have confirmed that changes in the diastolic closing pressure in the central retinal artery reflect the hydrostatic gradient between the eye and the heart. ${ }^{3}$

The brachial artery pressure was measured with the subject sitting, lying and standing. The IOP was measured with a Perkins tonometer. Once a reliable doppler signal had been obtained, the gate size and position were kept fixed and all subsequent measurements were taken with the probe position and angle repeated as closely as possible.

\section{Study 2: Intraocular Pressure}

A scleral suction cup apparatus (developed by Professor Hill, Department of Ophthalmology, Royal College of Surgeons of England) was used to raise the intraocular pressure. The cup was placed in the inferotemporal conjunctival fornix. The IOP and mean BP were measured as before, with the subject supine. Recordings were made through several cycles of raised IOP.

\section{Results}

Study 1: Posture

The results of measurements are shown in Figure 3 . The velocity of blood flow throughout diastole is higher when lying than when either sitting or standing.

\section{Study 2: Intraocular Pressure}

The results from two subjects are shown in Figure 4(a) and (b). The derived indices do not follow changes in IOP as markedly as in Study 1 . They are important, however, since this is the only experimental situation which discriminates between ocular and non-ocular blood flow. T(A)max most closely follows the transmural pressure changes.

\section{POSTURE VS VELOCITY}

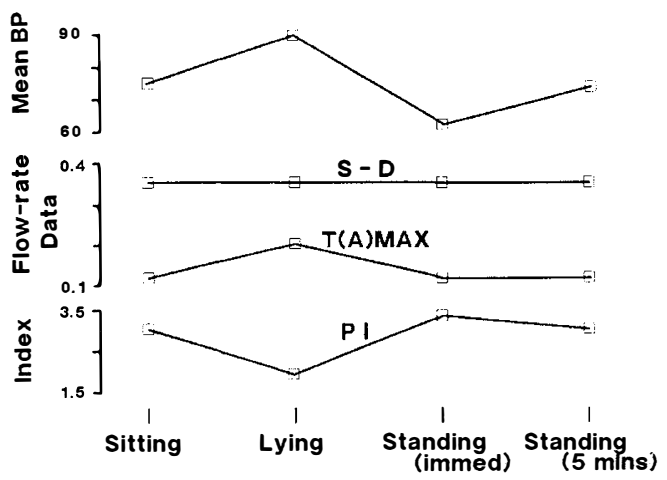

Fig. 3. Effect of changes in posture. Mean BP is the mean brachial artery pressure, less $22 \mathrm{mmHg}$ for the sitting and standing positions.

\section{Discussion}

The principle that a moving scatterer of sound alters the frequency of an interrogating sound pulse has been known for many years. In 1954 Kalmus ${ }^{4}$ proposed that the difference in transit time of ultrasonic pulses travelling upstream and downstream through a blood vessel between two probes could be used to assess the velocity of blood flow. Baldes ${ }^{4}$ demonstrated the technique experimentally in 1957. At about the same time Satomura ${ }^{4}$ demonstrated cardiac movement using backscattered ultrasonic pulses and one probe. The scatterers of sound in the blood stream were shown by Kato ${ }^{5}$ to be red blood cells.

In 1974 Till and Lessel ${ }^{6}$ demonstrated flow in branches of the ophthalmic artery. Other workers $^{7}$ have refined the techniques and 

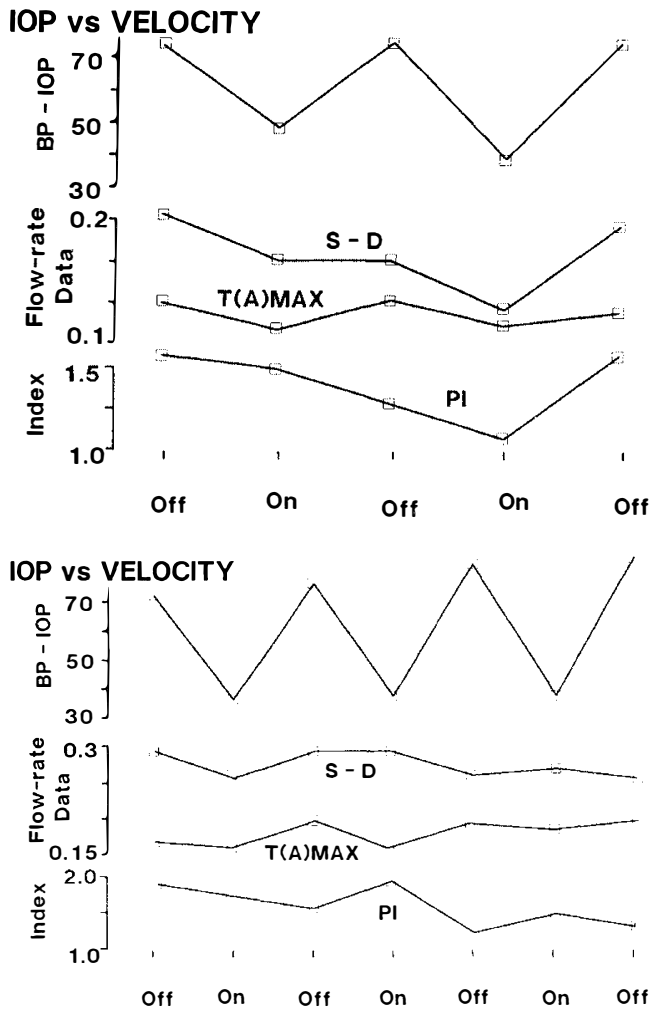

Fig. 4(a) and (b). Effect of induced changes in intraocular pressure. The results are from two subjects. 'On' and 'off' refer to whether or not suction was being applied to the scleral cup to raise IOP. 'BP-IOP' is the mean brachial artery pressure less the IOP.

applied them to the study of vascular disease in the orbit.

The preliminary studies reported here suggest that changes in blood pressure produce a corresponding fluctuation in both the RI and PI. Induced changes in IOP produce a less marked but recognisable change in some indices of blood flow rate, most obvious in the $\mathrm{T}(\mathrm{A}) \max$.

This technique may be of use in the study of vascular eye disease. It will be most useful where the disease process is unilateral so that the other eye can serve as a control, or in the study of the evolution of a disease, where repeated measurements can be made of the same vessel.

The authors wish to thank all their colleagues who participated in the IOP studies. Mr. Canning is a Friends of Moorfields Research Fellow.

\section{References}

${ }^{1}$ Hayreh SS: The ophthalmic artery. 3. Branches. Br J Ophthalmol 1962, 46: 212-47.

2 Bill A: Blood circulation and fluid dynamics in the eye. Physiol Rev 1975, 55: 383-417.

${ }^{3}$ Hague S and Hill DW: Postural changes in perfusion pressure and retinal arteriolar calibre. $\mathrm{Br} J$ Ophthalmol (in press).

${ }^{4}$ Wells PNT: History. In: de Vlieger M, Holmes JH, Kazner E, et al., eds, Handbook of Clinical Ultrasound. New York, John Wiley \& Sons; 1978, 3-13.

${ }^{5}$ Reid JM: principles of doppler ultrasound. In: de Vlieger M, Holmes JH, Kazner E, et al., eds, Handbook of Clinical Ultrasound. New York, John Wiley \& Sons; 1978, 81-90.

${ }^{6}$ Till $P$ and Lessel MR: Doppler techniques. In: de Vlieger M, Holmes JH, Kazner E, et al., eds, Handbook of Clinical Ultrasound. New York, John Wiley \& Sons; 1978, 905-17.

${ }^{7}$ Marmion VJ: Strategies in doppler ultrasound. Trans Ophthalmol Soc UK 1986, 105: 562-7. 